\title{
LA TUBERCULOSE EXTRA-GANGLIONNAIRE DE LA SPHERE O.R.L.
}

\section{EXTRANODAL ENT TUBERCULOSIS}

\author{
K. MIGHRI, I. LAHMAR, B. HAMMAMI, M. JLAIEL, A. MOUSSA, N. DRISS. \\ SERVICE O.R.L. CHU TAHAR SFAR MAHDIA
}

\begin{abstract}
RESUME
La tuberculose extra ganglionnaire est une pathologie rare en ORL. Elle pourrait poser des problèmes diagnostiques surtout avec la pathologie tumorale.

Nous rapportons 10 cas de localisations extra-ganglionnaires, colligés au service ORL de l'hôpital Tahar Sfar de Mahdia entre 1988 et 2004.

II s'agissait de 7 femmes et 3 hommes, âgés entre 19 et 77 ans (moyenne : 39 ans). La maladie a touché le cavum dans 3 cas, les glandes salivaires dans 3 cas, les amygdales palatines dans 2 cas et le larynx chez 2 patients.

Le diagnostic était dans tous les cas histo-pathologique. Tous nos patients ont reçu un traitement anti-tuberculeux. L'évolution était favorable avec un recul moyen de 11 mois.

Conclusion : La tuberculose extra-ganglionnaire en ORL pose surtout des problèmes diagnostiques. Son diagnostic est le plus souvent histo-pathologique. Son traitement repose sur la chimiothérapie anti-tuberculeuse.
\end{abstract}

\section{SUMMARY}

Extra-nodal tuberculosis is a rare in ENT practice. We report 10 cases of extra-ganglionic localizations of tuberculosis, treated in ENT department of the Tahar Sfar hospital of Mahdia between 1988 and 2004.

They were 7 women and 3 men, aged between 19 and 77 years (average: 39 years). The disease has touched the nasopharynx in 3 cases, the salivary glands in 3 cases, the tonsils in 2 cases and the larynx in 2 cases.

The diagnosis was in all cases histo-pathological. All our patients received an anti-tubercular treatment. The evolution was favorable with 11 months average outcome.

Conclusion: Extra-nodal tuberculosis in ENT poses especially diagnostic problems. Its treatment is based on anti-tubercular chemotherapy.

\section{INTRODUCTION}

La tuberculose a connu ces dernières décennies une grande élévation de son incidence en ORL, il s'agit d'une éventualité relativement rare, de traduction polymorphe et de localisations diverses.

Si la tuberculose ganglionnaire cervicale reste la plus fréquente en ORL, d'autres localisations plus rares peuvent se voir et posent parfois des difficultés diagnostiques.

\section{MATÉRIEL ET MÉTHODES}

II s'agit d'une étude rétrospective ayant porté sur 10 cas de tuberculose extra ganglionnaire, colligés au service ORL de l'hôpital Tahar Sfar de Mahdia, sur une période de 15 ans entre 1988 et 2004.

Pour chaque patient, un examen clinique complet a été réalisé, ayant comporté un interrogatoire (âge, sexe, antécédents de tuberculose, notion de contage, signes fonctionnels...) et un examen physique précisant les caractéristiques sémiologiques selon la localisation ainsi que la présence d'éventuelles adénopathies cervicales.

Certains examens complémentaires étaient demandés de façon systématique : une numération formule sanguine (NFS), une vitesse de sédimentation (VS) une intra dermoréaction à la tuberculine (IDR) et une radiographie de thorax. La certitude diagnostique était basée sur l'identification après biopsie d'un infiltrat granulomateux avec ou sans nécrose caséeuse. Pour les cas sans nécrose caséeuse, le contexte clinique et l'évolution favorable sous traitement anti-tuberculeux ont été en faveur du diagnostic de tuberculose. Tous nos patients ont reçu un traitement anti-tuberculeux.

\section{RÉSULTATS}

Nous avons colligé 10 cas de tuberculose extra-ganglionnaire pour un total de 62 cas de tuberculose de la sphère ORL, soit $16 \%$.

L'âge moyen de nos patients était de 39 ans, avec des extrêmes de 19 et 77 ans. II s'agissait de 7 femmes et 3 hommes.

Aucun patient n'avait de notion de contage ou d'antécédents de tuberculose.

Le délai moyen entre l'apparition de la symptomatologie et la prise en charge a varié entre 2 et 12 mois.

La localisation de la tuberculose était nasopharyngée dans 3 cas, parotidienne dans 2 cas, sub mandibulaire dans 1 cas, laryngée chez 2 patients et amygdalienne dans 2 cas. (fig. 1)

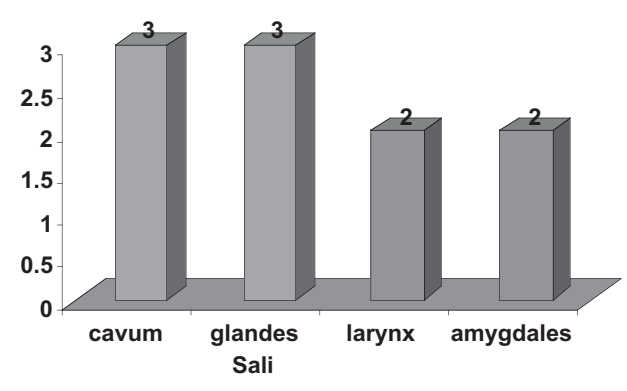

Fig $\mathrm{n}^{\circ} 1$ : Siège de la Tuberculose 
Cliniquement :

- 3 patients se plaignaient d'obstruction nasale associée à une rhinorrhée trainante. L'examen du cavum a objectivé un épaississemnt important des différentes parois.

- 2 patients ont consulté pour une tuméfaction parotidienne et un autre pour une tuméfaction sub-mandibulaire. Dans tous les cas cette tuméfaction était à l'examen, ferme, indolore et sans signes inflammatoires en regard.

- 2 patients ont consulté pour une dysphonie. A l'examen, il s'agissait de lésion bourgeonnante des cordes vocales.

- 2 patients ont consulté pour une odynophagie. L'examen a montré une ulcération amygdalienne unilatérale.

Des adénopathies cervicales ont été retrouvées chez 5 patients.

Sur le plan paraclinique :

- La vitesse de sédimentation était accélérée dans tous les cas.

- L'IDR à la tuberculine était positive dans 7 cas (phlycténulaire dans 1 cas)

- La radiographie du thorax a montré une atteinte pulmonaire concomitante dans 4 cas (fig. 2). Dans ces cas la recherche du BK dans le crachat était positive.

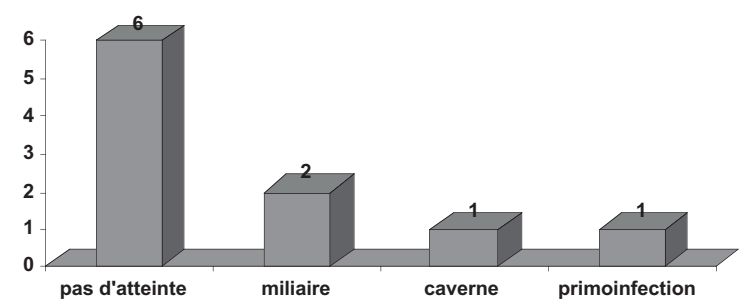

Fig. 2 : Atteinte pulmonaire concomitante

- Deux patients atteints de tuberculose parotidienne ont eu un scanner (fig. 3).

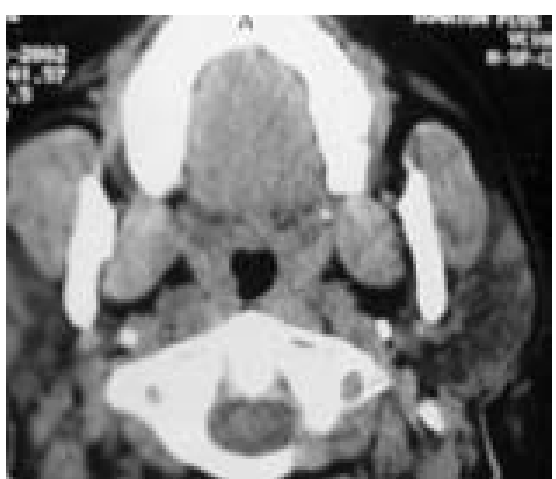

Fig. 3 : Scanner en coupe axiale : hypertrophie hétérogène de la glande parotide gauche

- Aucune cytoponction n'a été pratiquée.

Le diagnostic de la maladie était histo-pathologique dans tous les cas : sur étude de la biopsie pour les localisations nasopharyngées et laryngées, de la pièce d'exérèse chirurgicale (amygdalienne et parotidienne ou sub-mandibulaire). L'examen histo-pathologique a montré une inflammation gra- nulomateuse et giganto-cellulaire dans tous les cas. La nécrose caséeuse a été trouvée dans 8 cas.

Tous nos patients ont reçu un traitement médical à base d'anti- tuberculeux : quadruple (isoniazide, rifadine, streptomycine et pyazoline) pendant 2 mois suivi d'une bithérapie bihebdomadaire (isoniazide et rifadine) pendant 6 mois.

Les malades ayant une tuberculose des glandes salivaires ont bénéficié en plus du traitement chirurgical dans un but diagnostique (parotidectomie exo faciale dans deux cas et sub-mandibulectomie dans un cas), de même que les 2 patients ayant une localisation amygdalienne ont eu une amygdalectomie.

L'évolution était favorable dans 9cas, un patient a été perdu de vue.

Le recul moyen après la fin du traitement était de 11 mois.

\section{DISCUSSION}

La tuberculose reste toujours d'actualité avec, d'après les estimations de l'OMS, prés de 8 millions de personnes atteintes de tuberculose chaque année et 2 millions de décès. La recrudescence de la tuberculose est en partie expliquée par l'épidémie d'infection à VIH (1).

La tuberculose extra ganglionnaire de la sphère ORL est une pathologie rare, elle représente moins de $1 \%$ des localisations (2).

La tuberculose du rhinopharynx atteint surtout les sujets jeunes. Elle peut se manifester par des signes otologiques ou plus fréquemment par des adénopathies cervicales qui attirent l'attention vers le cavum (2).

Dans notre série, 2 patients sur 3 avaient des adénopathies cervicales et un seul avait une hypoacousie. Tous nos patients atteints de tuberculose du cavum avaient une localisation pulmonaire associée.

La tuberculose laryngée est rare et ne dépasse pas $1 \%$ de toutes les localisations $(3,4)$. Elle est souvent associée à une localisation pulmonaire ; environ $6 \%$ des patients n'ont pas d'atteinte pulmonaire évidente (3). Actuellement les localisations primitives sont de plus en plus fréquentes et par conséquent on risquerait de confondre la maladie avec d'autres pathologies tel qu'un carcinome (4).

La tuberculose laryngée peut se présenter sous plusieurs formes : ulcération, polype ou inflammation diffuse (3). Dans notre série, il s'agissait plutôt de lésions bourgeonnantes.

La tuberculose amygdalienne est une entité rare (5). Elle représente $13,6 \%$ des granulomatoses amygdaliennes selon l'étude de KAROLON (in 6 ).

L'amygdalectomie est réalisée à visée diagnostique et également thérapeutique en association au traitement médical (5).

En 1863, DEPAOLI (in 7) a rapporté le premier cas de tuberculose de la glande salivaire (parotide). Cette pathologie reste rare ; 200 cas ont été rapportés dans la littérature entre 1896 et 1987(in 8).

Le mécanisme de contamination glandulaire est discuté (9) ; deux voies ont été décrites : 
- voie retro-grade impliquant un foyer de contamination oropharyngé suivant un trajet ascendant et empruntant les canaux salivaires,

- voie hématogène ou lymphatique à point de départ pulmonaire.

La tuberculose glandulaire touche surtout le sujet jeune. Les 2 sexes sont atteints de la même façon $(7,9)$. L'état dentaire précaire est rapporté comme étant un facteur favorisant (7). La chirurgie constitue un moyen diagnostique $(7,10)$. Le traitement anti-tuberculeux est de principe $(10,11,12)$.

Plusieurs autres localisations de tuberculose extra-ganglionnaire de la sphère $O R L$ ont été rapportées dans la littérature comme (6):

- la tuberculose des cavités naso-sinusiennes : son incidence est estimée à $1 \%$ des tuberculoses de la tête et du cou.

- la tuberculose thyroïdienne, secondaire à la dissémination hématogène du germe : son incidence est estimée à 0,5\% des tuberculoses de la tête et du cou.

- la tuberculose auriculaire a été décrite pour la 1ère fois en $1883(3)$.
Le traitement de la tuberculose extra-ganglionnaire de la tête et du cou se base essentiellement sur l'isoniazide, la rifampicine, la pyrazinamide et la streptomycine actuellement remplacée par l'éthambutol $(6,7,13)$.

Certains auteurs prescrivent une chimiothérapie quadruple pendant 2 mois puis double pendant 4 mois. D'autres protocoles ont été décrits avec une quadruple thérapie pendant 2 mois, suivie à une triple thérapie pendant 7 mois (13). Le protocole adopté actuellement en Tunisie repose sur une quadruple thérapie quotidienne (isoniazide, rifadine, streptomycine et pyrazinamide) pendant 2 mois relayée par une bithérapie quotidienne ( isoniazide et rifadine) pendant 6 mois (14).

\section{CONCLUSION}

La tuberculose extra-ganglionnaire de la sphère ORL est une pathologie rare. Cliniquement elle pose des difficultés diagnostiques avec la pathologie néoplasique. Le diagnostic est essentiellement histo-pathologique.

Le traitement est basé sur les anti-tuberculeux. L'évolution est généralement favorable.

\section{REFERENCES}

1/ Chrétien J., Papillon F.

La tuberculose et les mycobactérioses à l'ere du Sida. Rev. Prat. (Paris), 1990, 40 (8): $709-714$

2/ Wookyng Moon, Moon Hee Han, Kee Jyun Chang et al. CT and MR imaging of head and Neck Tuberculosis Radiographies 1997; 17: 391-402

3/ Bhat Nalini, subramaniam Vinayak :

Tuberculosis in ear, nose, and throat practice : its presentation and diagnosis. American Journal of Otolaryngology-Head and Neck Medicine and Surgery ; 2006, 27:39-45

4/ Egeli E.,Oghan F., Alper M. et al

Epiglottic tuberculosis in a patient treated with steroids for addison's disease. Toroku J.Exp. Med ; 2003, 201 : 119-125.

5/ Yamamoto K.Iwata, F., Nakamura A. et al

Tonsillar tubercolsis associated with pulmonary and laryngeal foci Internal Medicine : August 2002, 41 (8) : 664-666.

6/ Legros M., J Mambie J., longnebray A. et al. La tuberculose en ORL

Journal français d'oto-rhino-laryngologie Novembre $1986 ; 35(9)$ : $\quad 427-431$

7/ Manipoud P., Rerolle S., Kemeny J.L. et al. Tuberculose de la glande sous- maxillaire Ann. Oto-laryng (Paris), 1993; 110: 482-484.

8/ A.Raji, M. Mahtar, M.Essaadi, et al La tubercolose parotidienne. A propos de six cas.

Med Mal infect ; 2002, 32 : 196-201.

9/ M'barek C., Sethom A.,Jhaider E. et al. Tuberculose primitive des glandes salivaires Maghreb Médical-volume Novembre 2000 ; 20 (352) : 390-391

10/ Aknin J., Disant F., H Morgon A.

La tuberculose de la parotide

Journal français d'oto-rhino-laryngologie, Décembre 1988 ; 37 (10) : 476-480

11/ Hunter D.C.,thomas J.M. Tuberculosis in the parotid region Br. J. Surg 1993, Vol 80 , Angust ; 1008

12/ Ubhi S.S., Neoptolemos J.P, Watkin D.F.L. Incidence and diagnosis of parotid gland tuberculosis in Asians in Leicester Br.J. Surg. 1988, vol 75, April, 313 13/ Grosset J. Bases bactériologiques du traitement de la tuberculose Rev. Prat. (Paris), $1990 ; 40$ (8) : 715-718

14/ Djebeniani R. : programme national de lutte antituberculeuse : guide technique 3ème édition ; 2004 : 40 